\title{
Frontal Alpha Asymmetry Moderated by Suicidal Ideation in Patients with Major Depressive Disorder: A Comparison with Healthy Individuals
}

\author{
Sang-Choong Roh', Ji Sun Kim², Sungkean Kim ${ }^{1,3}$, Yourim Kim ${ }^{1}$, Seung-Hwan Lee ${ }^{1,4}$ \\ ${ }^{1}$ Clinical Emotion and Cognition Research Laboratory, Inje University, Goyang, ${ }^{2}$ Department of Psychiatry, Soonchunhyang University Hospital, \\ Cheonan, ${ }^{3}$ Department of Biomedical Engineering, Hanyang University, Seoul, ${ }^{4}$ Department of Psychiatry, Ilsan Paik Hospital, Inje University \\ College of Medicine, Goyang, Korea
}

\begin{abstract}
Objective: Frontal alpha asymmetry (FAA) of electroencephalography (EEG) has been studied to differentiate patients with major depressive disorder (MDD) from healthy controls (HC). However, inconsistent results have been obtained thus far. Suicidal ideation (SI) has been known to alter frontal lobe activity, and could be an important covariate in FAA studies. This study aimed to explore the influence of FAA on the relationship among MDD patients with SI and without SI, and HC.

Methods: Sixty-seven patients with MDD (44 without and 23 with SI) and $60 \mathrm{HCs}$ were recruited. Resting state EEG was recorded with their eyes open, and FAA as a lateralized index of alpha power was calculated in the frontal brain region. Hamilton Rating Scale for Anxiety and Depression scores were estimated.

Results: FAA was higher (increased alpha power in the left frontal region) in the MDD group than in the HC group. The FAA was lower (reduced alpha power in the left frontal region) in MDD patients with SI than in MDD patients without SI. The severity of depression and anxiety symptoms were significantly correlated with FAA only in MDD patients with SI. SI moderated the effects of depressive symptom on FAA in the MDD group.

Conclusion: Our results suggest that $\mathrm{SI}$ is a clinically important moderator of frontal alpha asymmetry in patients with MDD.

KEY WORDS: Alpha asymmetry; Frontal alpha asymmetry; Major depressive disorder; Suicidal ideation; Quantitative electroencephalography.
\end{abstract}

\section{INTRODUCTION}

The prevalence of a lifetime major depressive episode is between $13 \%$ (Europe) and $17 \%$ (USA) [1-3]. It is also associated with suicide in about $15 \%$ of all patients with major depressive disorder (MDD) [4]. Suicidal ideation (SI) is a common and core symptom of MDD [5]. According to the Singapore Mental Health Study [6], the prevalence of SI among those with lifetime MDD was $43.6 \%$, wherein approximately $31.5 \%$ and $28.2 \%$ of individuals planned and attempted suicide respectively.

Received: October 10, 2018 / Revised: December 25, 2018 Accepted: January 23, 2019

Address for correspondence: Seung-Hwan Lee Department of Psychiatry, Inje University Ilsan Paik Hospital, Inje University College of Medicine, 170 Juhwa-ro, Ilsanseo-gu, Goyang 10380, Korea

E-mail: Ishpss@paik.ac.kr

ORCID: https://orcid.org/0000-0003-0305-3709
MDD has been known as a social and neuronal mental illness representing a complex mechanism of mental activity [7-9].

Enhanced alpha power of electroencephalography (EEG) has been known to reflect inhibition of activity in a region of the brain. The inverse relationship between alpha power and regional brain activity was proved in previous functional magnetic resonance imaging [10], near-infrared spectroscopy [11] and positron emission tomography studies [12]. In addition, several prior studies have reported significant differences in hemispheric asymmetry of frontal lobe alpha power (frontal alpha asymmetry [FAA]) between patients with MDD and healthy participants [13-15]. Henriques and Davidson [16] found that patients with MDD showed relatively less left-sided than right-sided activation in the mid-frontal region (i.e., increased alpha power in the left frontal region),

(ㄷ) This is an Open-Access article distributed under the terms of the Creative Commons Attribution Non-Commercial License (http://creativecommons.org/licenses/by-nc/4.0) which permits unrestricted non-commercial use, distribution, and reproduction in any medium, provided the original work is properly cited. 
whereas this activation was the opposite for healthy participants. Davidson [17] proposed that abnormal FAA is a trait marker of vulnerability for affective disorders or behaviors associated with decreased approach-related positive affect (i.e., reduced alpha in the left frontal region). However, a recent meta-analysis study by van der Vinne et al. [18] revealed a non-significant effect size and limited diagnostic value of FAA in MDD, which may lead to new discussions in the field of frontal alpha power.

Sex [18,19], symptom severity [18], and comorbidity [20] were considered as clinical covariates for FAA. Specifically, one meta-analysis study demonstrated that both depressive and anxious symptomatology show moderate relationships with relative right-sided frontal asymmetry, but comorbid studies show smaller average effects [20]. van der Vinne et al. [18] observed a three-way interaction of FAA, age, and depression severity in different sexes. A pattern of less relative left frontal activity was observed in women with high depressive severity than in those with low depressive severity, which is in contrast to results obtained in men [19]. Therefore, these previous results suggest that individual differences such as sex, symptom severity, and comorbidity should be considered while evaluating the relationship between MDD and FAA.

In addition, frontal lobe dysfunction in individuals with SI was repeatedly reported in previous studies $[21,22]$. Sublette et al. [23] highlighted that a relative hypometabolism in the right dorsolateral prefrontal cortex (DLPFC) and hypermetabolism in the left ventromedial re- gion are associated with SI. Graae et al. [24] reported that adolescents with a suicidal tendency had left-dominant alpha asymmetry while matched controls had right-dominant alpha asymmetry. Iosifescu et al. [5] found that frontal asymmetry of combined theta and alpha power was associated with worsening SI in patients with MDD during 4 weeks of antidepressant treatment. Recently, FAA, especially that of the low alpha band $(8-10 \mathrm{~Hz})$, was suggested to reflect suicidal behaviors in MDD patients [25]. However, the relationships among FAA, symptom severity, and SI have not been explored yet in patients with MDD.

Therefore, in this study, we aim to explore the FAA differences between MDD patients and healthy participants and to investigate the relationship between FAA and symptom severity in MDD patients with and without SI. We hypothesized that (1) FAA differs among MDD patients with and without $S I$ and healthy participants, (2) the symptom severity in MDD patients with and without $\mathrm{SI}$ is associated with alpha asymmetry, and (3) SI plays a role in the relationship between MDD and FAA.

\section{METHODS}

\section{Participants}

Sixty-seven patients with MDD (mean age, 38.67 years: 44 without and 23 with $\mathrm{SI}$ ) were recruited among the outpatients at the Department of Psychiatry in Inje University Ilsan Paik Hospital, Korea (Table 1). Sixty healthy controls

Table 1. ANOVA of the demographics, psychiatry rating scores, and FAA in MDD patients and HCs

\begin{tabular}{|c|c|c|c|c|c|c|}
\hline Variable & $\begin{array}{c}\text { MDD patients } \\
\text { without SI }(n=44)\end{array}$ & $\begin{array}{l}\text { MDD patients } \\
\text { with SI }(n=23)\end{array}$ & $\mathrm{HC}(\mathrm{n}=60)$ & $\digamma$ & $p$ & $\eta 2$ \\
\hline Age (yr) & $39.30 \pm 11.29$ & $37.48 \pm 9.33$ & $34.83 \pm 9.40$ & 2.538 & 0.083 & 0.039 \\
\hline Sex & & & & $0.692^{\mathrm{a}}$ & 0.708 & \\
\hline Male & $5(11.4)$ & $4(17.4)$ & $10(16.7)$ & & & \\
\hline Female & $39(88.6)$ & $19(82.6)$ & $50(83.3)$ & & & \\
\hline HAM-A & $24.91 \pm 5.03$ & $25.48 \pm 5.12$ & & 0.191 & 0.664 & 0.003 \\
\hline HAM-D & $29.27 \pm 6.54$ & $30.83 \pm 6.32$ & & 0.871 & 0.354 & 0.013 \\
\hline \multicolumn{7}{|l|}{ FAA } \\
\hline $\mathrm{FP} 1-\mathrm{FP} 2^{\dagger}$ & $4.87 \pm 16.05$ & $10.77 \pm 16.55$ & $-4.66 \pm 29.10$ & $4.398^{*}$ & 0.014 & 0.066 \\
\hline F3-F4 & $5.68 \pm 23.80$ & $4.83 \pm 23.05$ & $3.39 \pm 22.04$ & 0.132 & 0.877 & 0.002 \\
\hline $\mathrm{F} 7-\mathrm{F} 8^{\ddagger}$ & $26.17 \pm 13.02$ & $16.97 \pm 17.18$ & $10.65 \pm 21.82$ & $9.059^{* * *}$ & $<0.001$ & 0.127 \\
\hline
\end{tabular}

Values are presented as mean \pm standard deviation or number $(\%)$.

ANOVA, analysis of variance; FAA, frontal alpha asymmetry; MDD, major depressive disorder; HC, healty control; SI, suicidal ideation; HAM-A, Hamilton Rating Scale for Anxiety; HAM-D, Hamilton Rating Scale for Depression.

a By chi-square test; * $p<0.05, * * * p<0.001$.

Following least significant difference post-hoc analysis: ${ }^{\dagger}$ MDD patients with $\mathrm{SI}>\mathrm{HC}(p=0.23),{ }^{\ddagger}$ MDD patients without $\mathrm{SI}>\mathrm{HC}(p<0.001)$. 
(HC; mean age, 34.83 years) were recruited among social communities around the hospital through posters and local newspapers. The patients were diagnosed with MDD based on the Diagnostic and Statistical Manual of Mental Disorders fourth edition [26] by a psychiatrist. Patients with MDD who had any identifiable psychiatric illness such as psychotic disorder, panic disorder, obsessive-compulsive disorder, generalized anxiety disorder, social anxiety disorder, and post-traumatic stress disorder were excluded from our study. The diagnoses were established by a board-certified psychiatrist (SHL). All patients were drug naïve for at least 2 weeks when entering this study. Symptom severity was assessed by a psychologist using the Hamilton Rating Scale for Anxiety (HAM-A) and the Hamilton Rating Scale for Depression (HAM-D) [27]. The patients with SI were asked to evaluate whether they had concrete suicidal thoughts within the previous two years. The SI experienced during the ongoing depressive episode was considered positive. When individuals experienced SI in any previous depressive episodes, it was considered negative. Upon obtaining the results, the patients were divided into two groups according to the presence or absence of SI. HCs did not have any clinical diagnosis of psychiatric, neurological, and significant medical illness, and they did not have any previous history of psychiatric illness, and family history of psychiatric illness.

All participants were right-handed by the criterion of scoring above $80 \%$ on the Edinburgh Inventory Index [28]. All participants provided written informed consent after receiving a comprehensive explanation of the study protocol. This study was approved by the Institutional Review Board of Inje University Ilsan Paik Hospital (No. 2010-05-220).

\section{Measurement}

HAM-A: This scale assesses the severity of anxiety symptoms. It takes approximately 10 to 15 minutes to complete the interview and score the results. The scale consists of 14 items, each defined by a series of symptoms, and measures both psychic anxiety (mental agitation and psychological distress) and somatic anxiety (physical complaints related to anxiety). Each item is scored on a scale of 0 (not present) to 4 (severe), with a total score range of 0 to 56 , where $<17$ indicates mild, 18 to 24 mild to moderate, and 25 to 30 moderate to severe anxiety.

HAM-D: This scale assesses the severity of and change in depressive symptoms. It takes approximately 15 to 20 minutes to complete the interview and score the results. It consists of 17 items of the Likert scale, where eight items are scored on a 5-point scale, ranging from 0 (not present) to 4 (severe) and nine items are scored from 0 to 2 .

\section{EEG Recordings and Quantitative EEG Analyses}

The participants were seated in a dimly lit and soundattenuated room. The resting state EEG was recorded with the participants' eyes open and closed for 3 minutes each. The EEG signals were acquired using a NeuroScan SynAmps 2 amplifier (Compumedics, El Paso, TX, USA) with 62 surface electrodes (FP1, FPZ, FP2, AF3, AF4, F7, F5, F3, F1, FZ, F2, F4, F6, F8, FT7, FC5, FC3, FC1, FCZ, FC2, FC4, FC6, FT8, T7, C5, C3, C1, CZ, C2, C4, C6, T8, TP7, CP5, CP3, CP1, CPZ, CP2, CP4, CP6, TP8, P7, P5, P3, P1, PZ, P2, P4, P6, P8, PO7, PO5, PO3, POZ, PO4, PO6, PO8, CB1, O1, OZ, O2, and CB2) mounted on a Quik-Cap (Compumedics) according to the extended international 10-20 placement scheme. The ground electrode was placed on the forehead and the reference electrode was predefined in the cap between $\mathrm{Cz}$ and $\mathrm{CPz}$. The vertical electrooculogram (EOG) was recorded using bipolar electrodes; one was located above the right eye and one below it. The horizontal EOG was recorded at the outer canthus of each eye. The impedance of the electrodes was maintained at less than $5 \mathrm{k} \Omega$.

EEG data were obtained using a 0.1 to $100 \mathrm{~Hz}$ band-pass filter at a sampling rate of $1,000 \mathrm{~Hz}$ and were initially processed using Scan 4.3 (Compumedics). Eye movements and blink artifacts were visually screened and eliminated by a trained technician with no prior information regarding the origin of the data. In this study, we only analyzed the resting EEG data with eyes open. Previous studies have shown no difference between eyesopen and eyes-closed conditions in EEG FAA $[16,19,29,30]$. In addition, a neurofeedback study with EEG FAA demonstrated that the change in alpha asymmetric brain activity pattern was only found in the eyes-open condition, suggesting that the training effect for alpha asymmetry should be measured in the eyes-open condition [31]. Although the eyes-closed condition is more widely used, the eyes-open condition is also appropriate for alpha asymmetry. 
EEG data were divided into epochs with a length of $\sim 2$ seconds (2,048 points) and the epochs with signals exceeding $\pm 80 \mu \mathrm{V}$ on any channel were excluded from further analysis [32]. Thirty epochs ( 60 seconds) were prepared for each subject. Fast Fourier transformation was performed on 62 electrode channels divided into five frequency bands: delta $(1-4 \mathrm{~Hz})$, theta $(4-8 \mathrm{~Hz})$, alpha (8$12 \mathrm{~Hz})$, beta $(12-30 \mathrm{~Hz})$, and gamma $(30-50 \mathrm{~Hz})[33]$. The absolute power of each channel was then calculated. Based on previous literature [32-35], we considered three paired sites of interest: pre-frontal (Fp1 - Fp2), mid-frontal (F3-F4), and lateral-frontal (F7-F8) area.

To analyze FAA, we used the lateral asymmetry index [36]. This index was determined by comparing the corresponding frequency band percentages for the left and right hemispheres. It was computed by dividing the differences between the two hemispheres by their sum, i.e., $\mathrm{A}=$ $\left(P_{\text {left }}-P_{\text {right }}\right) /\left(P_{\text {left }}+P_{\text {right }}\right) \times 100$, where $P_{\text {left }}$ and $P_{\text {right }}$ are the absolute powers of the corresponding frequency band in the appropriate brain electrodes. Thus, an index of zero indicates equivalent activity in both the hemispheres. A higher FAA indicates dominant brain activity in the right hemisphere, whereas a lower FAA indicates dominant brain activity in the left hemisphere.

\section{Statistical Analysis}

Group comparisons of demographic data were performed using one-way analysis of variance (ANOVA). The independent $t$ test was used to assess differences in symptom scales (HAM-A and HAM-D) between MDD patients with and without SI. The chi-square test was used to assess differences in sex distribution among groups. For FAA, one-way ANOVA was performed to assess differences among the three groups. The variables showing sig- nificant differences were further analyzed with post-hoc pairwise comparison using least significant difference (LSD). Spearman's correlation analysis was performed to evaluate the relationship between the FAA and symptom scales in patients with MDD. The bootstrap resampling technique ( $n=5,000)$ was used to correct multiple correlations. Using the significance of correlation among the sites of interest, we completed a three-step hierarchical regression to explore the interaction effects of HAM-A and HAM-D by SI on FAA (F3-F4 and F7-F8). In the first stage, we controlled for the effects of demographic variables in the regression model. In the second stage, we input HAM-A and SI to control for the main effects on FAA (F3 -F4) and input HAM-D and SI to control for the main effects on FAA (F7 - F8). In the third stage, we analyzed the interaction effect of HAM-A by SI on FAA (F3-F4) and HAM-D by SI on FAA (F7 - F8). The significance level was set at $p<0.05$. Statistical analyses were performed using SPSS software (version 18.0; IBM Corp., Armonk, NY, USA). Hayes and Preacher's [37] bootstrapping macro designed for SPSS was also performed to verify the interaction effects.

\section{RESULTS}

\section{Symptom Severity and Alpha Asymmetry}

Symptom severity ranged from mild to severe in MDD patients with and without SI and no significant group difference in symptom scales was found between the two groups (Table 1). There was a significant difference among the groups in FAA of the pre-frontal area (Fp1 - Fp2), [ $F(2$, $124)=4.398, p=0.014, \eta 2=0.066]$; post-hoc analysis revealed that the FAA of MDD patients with SI was significantly higher than that of $\mathrm{HCs}(p=0.23)$. However, no

Table 2. Spearman's correlation (rho) between clinical symptoms and FAA in all MDD patients and MDD patients with SI

\begin{tabular}{|c|c|c|c|c|c|c|c|c|}
\hline \multirow{3}{*}{ FAA } & \multicolumn{4}{|c|}{ All MDD patients $(n=67)$} & \multicolumn{4}{|c|}{ MDD patients with $\mathrm{SI}(\mathrm{n}=23)$} \\
\hline & \multicolumn{2}{|c|}{ HAM-A } & \multicolumn{2}{|c|}{ HAM-D } & \multicolumn{2}{|c|}{ HAM-A } & \multicolumn{2}{|c|}{ HAM-D } \\
\hline & $r$ & $p$ & $r$ & $p$ & $r$ & $p$ & $r$ & $p$ \\
\hline FP1-FP2 & 0.023 & 0.855 & 0.008 & 0.950 & 0.029 & 0.895 & -0.200 & 0.360 \\
\hline F3-F4 & 0.190 & 0.123 & 0.015 & 0.907 & $0.532 * *$ & 0.009 & -0.147 & 0.503 \\
\hline F7-F8 & -0.101 & 0.418 & $-0.322^{* *}$ & 0.008 & -0.017 & 0.939 & $-0.518^{*}$ & 0.011 \\
\hline
\end{tabular}

FAA, frontal alpha asymmetry; MDD, major depressive disorder; SI, suicidal ideation; HAM-A, Hamilton Rating Scale for Anxiety; HAM-D, Hamilton Rating Scale for Depression.

${ }^{*} p<0.05,{ }^{* *} p<0.01$. 
significant difference was found between MDD without SI and HCs. There was also a significant difference among the groups in FAA of the lateral-frontal area $(\mathrm{F} 7-\mathrm{F} 8)[F(2$, $124)=9.059, p<0.001, \eta 2=0.127] ;$ post - hoc analysis revealed that the FAA of MDD without SI was significantly higher than that of HCs $(p<0.001)$, and the FAA of MDD without $\mathrm{SI}$ was marginally significantly higher than that of MDD patients with SI $(p=0.054)$. However, no meaningful difference was found between MDD patients with $\mathrm{SI}$ and HC. No significant difference was found in FAA (F3 $-F 4$ ) among the groups.

When all MDD patients were compared to $\mathrm{HCs}$, significant differences were found in FAA (FP1 - FP2: $6.89 \pm$ 16.34 vs. $-4.66 \pm 29.10, p=0.008 ;$ F7 - F8: $23.01 \pm$ 15.10 vs. $10.65 \pm 21.82, p<0.001$ ).

ANOVA of the demographics, psychiatry rating scales, and FAA in MDD patients with and without $\mathrm{SI}$ and $\mathrm{HCs}$ are presented in Table 1.

\section{FAA and Its Correlation with Symptom Severity Scores}

Table 2 shows the correlation between clinical variables (HAM-A and HAM-D) and FAA in all MDD patients $(n=67)$, and MDD patients with $\mathrm{SI}(\mathrm{n}=23)$. In all MDD patients, there was a significant correlation $(r=-0.322$, $p=0.008$ ) between HAM-D and FAA (F7-F8). Significant correlations were found in FAA of $\mathrm{F} 3-\mathrm{F} 4$ and F7 - F8 areas in MDD patients with SI. The FAA (F3-F4) was positively correlated with the HAM-A score $(r=$ $0.532, p=0.009$ ), which indicates that higher alpha power in the left mid-frontal region reflects higher anxiety lev- els and decreased brain activity levels in that region. The FAA (F7 - F8) was negatively correlated with HAM-D $(r=-0.518, p=0.011)$, which indicates that lower alpha power in the left lateral frontal region reflects higher depression levels and increased brain activity levels in that region. The scatterplot of the relationship between the FAA and clinical variables (HAM-A and HAM-D) in the mid-frontal and the lateral frontal areas in MDD patients with $\mathrm{SI}$ are presented in Figure 1. However, no significant correlation between FAA and clinical variables in MDD patients without SI was found. When the data were re-analyzed without including male participant data, significant results were still obtained.

Table 3. Hierarchical regression analysis of the interaction effects of HAM-D by SI on FAA (F7-F8) in MDD patients $(n=67)$

\begin{tabular}{lrrrrr}
\hline Predictor & $\begin{array}{c}\text { Standardized } \\
\beta\end{array}$ & $\begin{array}{c}\text { Standard } \\
\text { error }\end{array}$ & $\mathrm{T}$ & $p$ & $\Delta \mathrm{R} 2$ \\
\hline Constant & & 15.292 & 1.327 & 0.189 & 0.030 \\
Sex & 0.007 & 5.905 & 0.050 & 0.961 & \\
Age & 0.162 & 0.212 & 1.085 & 0.282 & \\
Alcohol & 0.085 & 5.452 & 0.529 & 0.599 & \\
Smoking & 0.002 & 6.207 & 0.015 & 0.988 & \\
Marriage & -0.187 & 3.389 & -1.204 & 0.233 & 0.150 \\
HAM-D & -0.251 & 0.284 & $-2.066^{*}$ & 0.043 & \\
SI & 0.319 & 4.310 & $2.337^{*}$ & 0.023 & \\
HAM-D $\times$ SI & 1.292 & 0.588 & $1.912^{\dagger}$ & 0.061 & 0.049 \\
\hline
\end{tabular}

HAM-D, Hamilton Rating Scale for Depression; SI, suicidal ideation; FAA, frontal alpha asymmetry; MDD, major depressive disorder. ${ }^{*} p<0.05,{ }^{\dagger} p<0.07$.
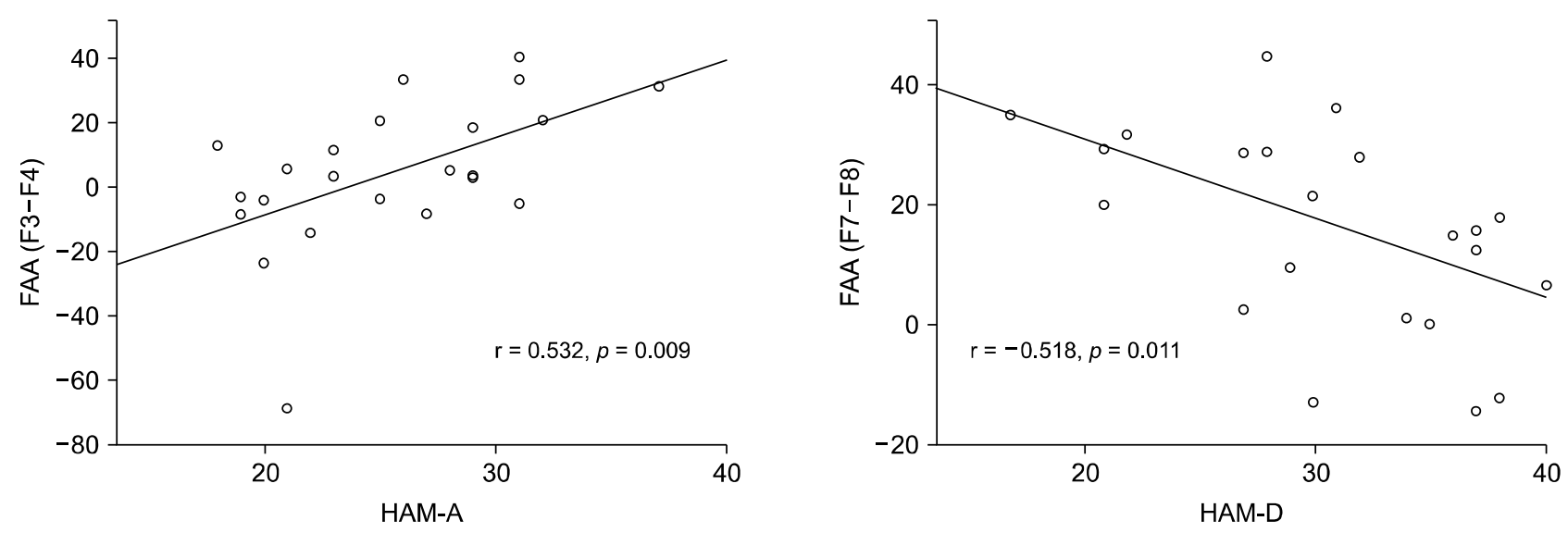

Fig. 1. Spearman's correlations between frontal alpha asymmetry (FAA) and symptom severity score in patients with major depressive disorder with suicidal ideation $(n=23)$. HAM-A, Hamilton Rating Scale for Anxiety; HAM-D, Hamilton Rating Scale for Depression. 
Table 4. Interaction effect of HAM-D on FAA (F7 - F8) at values of the moderator(s)

\begin{tabular}{lccccc}
\hline \multicolumn{1}{c}{ Variable } & Effect & Boot SE & $\mathrm{t}$ & $\mathrm{p}$ & $95 \% \mathrm{Cl}$ \\
\hline MDD with SI & -1.3262 & 0.4904 & -2.7043 & 0.0090 & -2.3085 to -0.3438 \\
MDD without SI & -0.2663 & 0.3526 & -0.7553 & 0.4532 & -0.9728 to 0.4401 \\
\hline
\end{tabular}

Number of bootstrap samples for bias corrected bootstrap confidence intervals $=5,000$.

HAM-D, Hamilton Rating Scale for Depression; SE, standard error; Cl, confidence interval; SI, suicidal ideation.

A

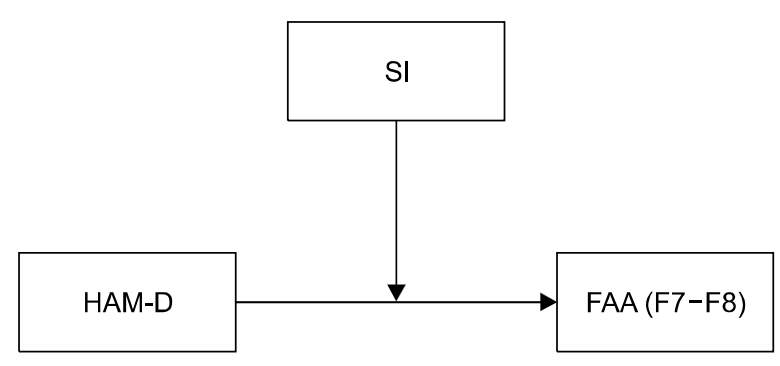

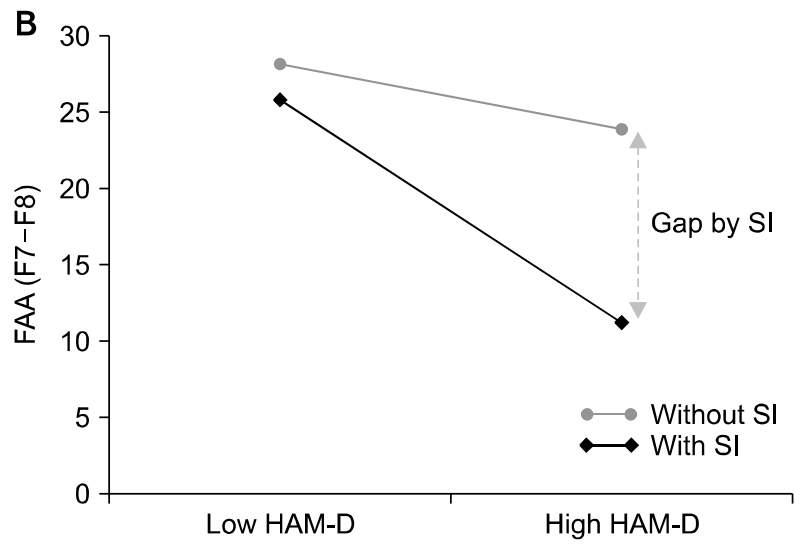

Fig. 2. (A) Moderation effect of suicidal ideation (SI) on the Hamilton Rating Scale for Depression (HAM-D) and the frontal alpha asymmetry (FAA) at F7-F8. (B) Severe depressive symptom causes reduced FAA (F7-F8), which reflects reduced alpha power in the left frontal region. This phenomenon was only significant in in patients with major depressive disorder with suicidal ideation $(n=23)$.

\section{Interaction effect of HAM-D by SI}

Table 3 illustrates the hierarchical regression analysis for the interaction effect of HAM-D by SI on FAA (F7 - F8) in MDD patients. No demographic variable had a significant effect on FAA (F7-F8). With demographic variables controlled for in step 2, the HAM-D showed a significant main effect on FAA (F7-F8) ( $\beta=-0.251, p=$ 0.043 ) and SI showed a significant main effect on FAA (F7 -F8) ( $\beta=0.319, p=0.023)$. When main effects of these variables were controlled for in step 3 , the interaction effect of HAM-D by SI showed a marginal significance with $4.9 \%$ of additional $\mathrm{R}$ square $(\beta=1.292, p=0.061)$. Table 4 illustrates the verification of the interaction effect in MDD patients with SI $(\beta=-1.3262, p=0.009)$; zero does not appear in the $95 \%$ confidence interval that lies between -2.3085 and -0.3438 . In Figure 2, the graphs represent $(A)$ a moderation effect of $S I$ between HAM-D and FAA (F7-F8), and (B) a significant difference between low HAM-D and high HAM-D scores in MDD patients with SI; no significant difference was observed in the group without SI. No interaction effect was found on FAA (F3-F4). When the data were re-analyzed without including male participant data, the results still showed significance.

\section{DISCUSSION}

This study aimed to explore the FAA among MDD patients with SI, those without SI, and HC. The results revealed that significant FAA differences exist between MDD patients with SI and HCs at FP1 - FP2 and between MDD patients without $\mathrm{SI}$ and $\mathrm{HCs}$ at F7-F8. Furthermore, HAM-A and HAM-D scores were positively and negatively significantly correlated, respectively, with FAA (F3-F4 and F7-F8) in the MDD patients with SI, and $\mathrm{SI}$ moderates the effects of depressive symptom on the FAA (F7-F8) in MDD patients.

Our findings showed that regardless of the presence of $\mathrm{SI}$, patients with MDD have a higher FAA than that of HCs. This implies that the right frontal activity increased in patients with MDD. Previous studies have repeatedly reported differences in FAA between MDD and HCs $[16,18,38,39]$. Our results are consistent with those of previous studies, which showed that compared with the right frontal lobe, patients with MDD have relative hypoactivation in the left frontal lobe, while HCs have relative 
hyperactivation in the left frontal lobe $[16,40]$.

Furthermore, our study reported a marginally significantly higher FAA in MDD patients without $\mathrm{SI}$ than in those with $\mathrm{SI}$ in the lateral-frontal area (F7-F8). This indicates that activity is more skewed in the left side of the brain in MDD patients with SI than in MDD patients without SI. Pu et al. [11] reported significantly smaller hemodynamic changes in the right DLPFC, orbitofrontal cortex, and right frontopolar cortex in MDD patients with SI. Moreover, Sublette et al. [23] observed a significantly reduced glucose metabolism in the right DLPFC in MDD patients who attempted suicide than in those who did not. The results from these previous studies are consistent with ours as they indicate that MDD patients with SI have left skewed frontal activity rather than right frontal activity. Our results suggest that FAA is influenced by $\mathrm{SI}$ in patients with MDD, and SI could be a significant covariate of FAA.

HAM-A scores showed a significant positive correlation with the FAA only in MDD patients with SI. Our results suggest that higher HAM-A scores are associated with relatively right skewed frontal activity. This result is in line with those of several previous studies, which showed that increased relative EEG activity in the right frontal brain regions is associated with anxiety symptoms and anxiety disorders $[41,42]$. Using FAA, Nitschke et al. [43] compared brain activity between anxious apprehension (worry) and anxious arousal (somatic anxiety) participants, and reported that the anxious arousal group showed more right than left frontal brain activity. Our results indicated that MDD patients with higher anxiety and arousal levels have right skewed frontal activity. Moreover, because anxiety could be one of the clinical factors associated with the presence of SI [44], the FAA could be closely associated with the presence of anxiety $[45,46]$.

Interestingly, the HAM-D score showed a significant negative correlation with the FAA in MDD patients with SI. Our results suggest that higher HAM-D scores cause relatively left skewed frontal activity. In fact, previous studies did not obtain consistent results on the relationship between FAA and depression severity. Thibodeau et al. [20] also examined this in their meta-analysis, but failed to obtain any conclusive findings. Furthermore, Stewart et al. [19] showed that a pattern of less relative left frontal activity was observed in women with ongoing high depressive severity than in women with low depressive severity, which in contrast to the results obtained in men. van der Vinne et al. [18] also observed a significant three-way interaction for sex $x$ age $x$ depression severity in the patients with depression. They noted that the right-sided FAA (left skewed frontal activity) is higher in severely depressed women aged 53 years and older, in contrast to a relatively more left-sided FAA in severely depressed men of the same age. Thus, further investigation is required on this topic and our results suggest that SI might be an important factor for future research.

Furthermore, in this study, SI was investigated to evaluate its role in the relationship between depressive symptom severity and FAA. Even though clinical variables, including age and sex, were considered as covariates, the interaction effect of HAM-D with SI was found in patients with MDD. This result implies that SI moderates the effects of depressive symptom severity on FAA. A significant FAA difference between low and high HAM-D was found only in MDD patients with SI, whereas no significance was observed in MDD patients without SI. In this study, the $p$ value of 0.61 obtained in the hierarchical interaction effect analysis seems marginal. However, as Selvin [47] strongly suggested, an increase in the level of significance is usually required to test the effect of an interaction, otherwise, erroneous elimination of interaction effects could substantially disrupt the validity the conclusions obtained.

It is important to note that this study had several limitations. First, the sample size of MDD patients with SI was relatively smaller than that of other groups. Second, the number of male participants was relatively small to fully explore the effect of sex. Despite the above limitations, the reported results provide a starting point for future FAA studies, indicating the importance of $\mathrm{SI}$ as a meaningful covariate.

Our results suggest that a difference in FAA exists between patients with MDD and HCs. The FAA could be associated with depressive symptom severity in MDD patients with SI. In addition, SI moderates the effects of depressive symptom on FAA. Our results suggest that SI should be considered as a moderator in future FAA studies.

\section{- Acknowledgments}

This work was supported by the 2017 creative research program of Inje University, and also supported by a grant from the Korea Science and Engineering Foundation 
(KOSEF), funded by the Korean government (NRF-2018 R1A2A2A05018505).

\section{Conflicts of Interest}

No potential conflict of interest relevant to this article was reported.

\section{Author Contributions}

Conceptualization: Sang-Choong Roh, Seung-Hwan Lee. Data acquisition: Sang-Choong Roh, Ji Sun Kim. Formal analysis: Sang-Choong Roh, Ji Sun Kim, Sungkean Kim. Funding: Seung-Hwan Lee. Supervision: Seung-Hwan Lee. Writing-original draft: Sang-Choog Roh. Writing-review \& editing: Yourim Kim, Seung-Hwan Lee.

\section{ORCID}

Sang-Choong Roh https://orcid.org/0000-0001-6809-3758 Ji Sun Kim https://orcid.org/0000-0003-2472-4591 Sungkean Kim https://orcid.org/0000-0003-4649-6112 Yourim Kim https://orcid.org/0000-0002-2809-8877 Seung-Hwan Lee https://orcid.org/0000-0003-0305-3709

\section{REFERENCES}

1. Alonso J, Angermeyer MC, Bernert S, Bruffaerts R, Brugha TS, Bryson $\mathrm{H}$, et al. Prevalence of mental disorders in Europe: results from the European Study of the Epidemiology of Mental Disorders (ESEMeD) project. Acta Psychiatr Scand Suppl 2004; (420):21-27.

2. Kessler RC, Berglund P, Demler O, Jin R, Merikangas KR, Walters EE. Lifetime prevalence and age-of-onset distributions of DSM-IV disorders in the National Comorbidity Survey Replication. Arch Gen Psychiatry 2005;62:593-602.

3. Murray CJ, Lopez AD. Alternative projections of mortality and disability by cause 1990-2020: Global Burden of Disease Study. Lancet 1997;349:1498-1504.

4. American Psychiatric Association. Diagnostic and statistical manual of mental disorders: DSM-IV-TR. 4th ed; 10th revision. Washington, DC:American Psychiatric Association;2000.

5. Iosifescu DV, Greenwald S, Devlin P, Perlis RH, Denninger JW, Alpert JE, et al. Pretreatment frontal EEG and changes in suicidal ideation during SSRI treatment in major depressive disorder. Acta Psychiatr Scand 2008;117:271-276.

6. Subramaniam M, Abdin E, Seow EL, Picco L, Vaingankar JA, Chong SA. Suicidal ideation, suicidal plan and suicidal attempts among those with major depressive disorder. Ann Acad Med Singapore 2014;43:412-421.

7. Jeon H, Lee SH. From neurons to social beings: short review of the mirror neuron system research and its socio-psychological and psychiatric implications. Clin Psychopharmacol
Neurosci 2018;16:18-31.

8. Jung $\mathrm{W}$, Jang $\mathrm{KI}$, Lee $\mathrm{SH}$. Heart and brain interaction of psychiatric illness: a review focused on heart rate variability, cognitive function, and quantitative electroencephalography. Clin Psychopharmacol Neurosci 2019;17:459-474.

9. De Berardis D, Olivieri L, Rapini G, Di Natale S, Serroni N, Fornaro $\mathrm{M}$, et al. Alexithymia, suicide ideation and homocysteine levels in drug naïve patients with major depression: a study in the "Real World" clinical practice. Clin Psychopharmacol Neurosci 2019;17:318-322.

10. Goldman RI, Stern JM, Engel J Jr, Cohen MS. Simultaneous EEG and FMRI of the alpha rhythm. Neuroreport 2002;13: 2487-2492.

11. Pu S, Nakagome K, Yamada T, Yokoyama K, Matsumura H, Yamada S, et al. Suicidal ideation is associated with reduced prefrontal activation during a verbal fluency task in patients with major depressive disorder. J Affect Disord 2015;181: 9-17.

12. Oakes TR, Pizzagalli DA, Hendrick AM, Horras KA, Larson $\mathrm{CL}$, Abercrombie $\mathrm{HC}$, et al. Functional coupling of simultaneous electrical and metabolic activity in the human brain. Hum Brain Mapp 2004;21:257-270.

13. Davidson RJ, Schaffer CE, Saron C. Effects of lateralized presentations of faces on self-reports of emotion and EEG asymmetry in depressed and non-depressed subjects. Psychophysiology 1985;22:353-364.

14. Tucker DM, Stenslie CE, Roth RS, Shearer SL. Right frontal lobe activation and right hemisphere performance. Decrement during a depressed mood. Arch Gen Psychiatry 1981; 38:169-174.

15. Tomarken AJ, Davidson RJ, Henriques JB. Resting frontal brain asymmetry predicts affective responses to films. J Pers Soc Psychol 1990;59:791-801.

16. Henriques JB, Davidson RJ. Left frontal hypoactivation in depression. J Abnorm Psychol 1991;100:535-545.

17. Davidson RJ. Anterior cerebral asymmetry and the nature of emotion. Brain Cogn 1992;20:125-151.

18. van der Vinne N, Vollebregt MA, van Putten MJAM, Arns M. Frontal alpha asymmetry as a diagnostic marker in depression: fact or fiction? A meta-analysis. Neuroimage Clin 2017; 16:79-87.

19. Stewart JL, Bismark AW, Towers DN, Coan JA, Allen JJ. Resting frontal EEG asymmetry as an endophenotype for depression risk: sex-specific patterns of frontal brain asymmetry. I Abnorm Psychol 2010;119:502-512.

20. Thibodeau R, Jorgensen RS, Kim S. Depression, anxiety, and resting frontal EEG asymmetry: a meta-analytic review. I Abnorm Psychol 2006; 115:715-729.

21. Richard-Devantoy S, Berlim MT, Jollant F. A meta-analysis of neuropsychological markers of vulnerability to suicidal behavior in mood disorders. Psychol Med 2014;44:1663-1673.

22. van Heeringen $K$, Wu GR, Vervaet $M$, Vanderhasselt MA, Baeken C. Decreased resting state metabolic activity in fronto- 
polar and parietal brain regions is associated with suicide plans in depressed individuals. J Psychiatr Res 2017;84:243248.

23. Sublette ME, Milak MS, Galfalvy HC, Oquendo MA, Malone KM, Mann JJ. Regional brain glucose uptake distinguishes suicide attempters from non-attempters in major depression. Arch Suicide Res 2013;17:434-447.

24. Graae F, Tenke C, Bruder G, Rotheram MJ, Piacentini J, Castro-Blanco D, et al. Abnormality of EEG alpha asymmetry in female adolescent suicide attempters. Biol Psychiatry 1996; 40:706-713.

25. Park Y, Jung W, Kim S, Jeon H, Lee SH. Frontal alpha asymmetry correlates with suicidal behavior in major depressive disorder. Clin Psychopharmaco/ Neurosci 2018. http://www. cpn.or.kr/journal/view.html?uid=825\&vmd=Full. [Epub ahead of print

26. American Psychiatric Association. Diagnostic and statistical manual of mental disorders: DSM-IV. 4th ed. Washington: American Psychiatric Association; 1994.

27. Hamilton M. A rating scale for depression. J Neurol Neurosurg Psychiatry 1960;23:56-62.

28. Oldfield RC. The assessment and analysis of handedness: the Edinburgh inventory. Neuropsychologia 1971;9:97-113.

29. Reid SA, Duke LM, Allen JJ. Resting frontal electroencephalographic asymmetry in depression: inconsistencies suggest the need to identify mediating factors. Psychophysiology 1998; 35:389-404.

30. Gordon E, Palmer DM, Cooper N. EEG alpha asymmetry in schizophrenia, depression, PTSD, panic disorder, ADHD and conduct disorder. Clin EEG Neurosci 2010;41:178-183.

31. Choi SW, Chi SE, Chung SY, Kim JW, Ahn CY, Kim HT. Is a/pha wave neurofeedback effective with randomized clinical trials in depression? A pilot study. Neuropsychobiology 2011; 63:43-51.

32. Jacobs GD, Snyder D. Frontal brain asymmetry predicts affective style in men. Behav Neurosci 1996;110:3-6.

33. Kim JS, Lee SH, Park G, Kim S, Bae SM, Kim DW, et al. Clinical implications of quantitative electroencephalography and current source density in patients with Alzheimer's disease. Brain Topogr 2012;25:461-474.

34. Carvalho JP, Hopko DR. Behavioral theory of depression: reinforcement as a mediating variable between avoidance and depression. J Behav Ther Exp Psychiatry 2011;42:154-162.

35. Gold C, Fachner J, Erkkilä J. Validity and reliability of electro- encephalographic frontal alpha asymmetry and frontal midline theta as biomarkers for depression. Scand J Psychol 2013; 54:118-126.

36. Jin $\mathrm{SH}, \mathrm{Na} \mathrm{SH}, \mathrm{Kim} \mathrm{SY}, \mathrm{Ham} \mathrm{BJ}$, Lee $\mathrm{DH}$, Lee JH, et al. Hemispheric laterality and dimensional complexity in schizophrenia under sound and light stimulation. Int J Psychophysiol 2003;49:1-15.

37. Hayes AF, Preacher KJ. Statistical mediation analysis with a multicategorical independent variable. Br JMath Stat Psychol 2014;67:451-470.

38. Henriques JB, Davidson RJ. Regional brain electrical asymmetries discriminate between previously depressed and healthy control subjects. J Abnorm Psychol 1990;99:22-31.

39. Debener S, Beauducel A, Nessler D, Brocke B, Heilemann H, Kayser J. Is resting anterior EEG alpha asymmetry a trait marker for depression? Findings for healthy adults and clinically depressed patients. Neuropsychobiology 2000;41:31-37.

40. Gotilb IH. EEG alpha asymmetry, depression, and cognitive functioning. Cogn Emot 1998;12:449-478.

41. Bruder GE, Fong R, Tenke CE, Leite P, Towey JP, Stewart JE, et al. Regional brain asymmetries in major depression with or without an anxiety disorder: a quantitative electroencephalographic study. Biol Psychiatry 1997;41:939-948.

42. Wiedemann G, Pauli P, Dengler W, Lutzenberger W, Birbaumer N, Buchkremer G. Frontal brain asymmetry as a biological substrate of emotions in patients with panic disorders. Arch Gen Psychiatry 1999;56:78-84.

43. Nitschke JB, Heller W, Palmieri PA, Miller GA. Contrasting patterns of brain activity in anxious apprehension and anxious arousal. Psychophysiology 1999;36:628-637.

44. Perlis RH, Beasley CM Jr, Wines JD Jr, Tamura RN, Cusin C, Shear D, et al. Treatment-associated suicidal ideation and adverse effects in an open, multicenter trial of fluoxetine for major depressive episodes. Psychother Psychosom 2007;76:4046.

45. Davidson RJ. Anterior electrophysiological asymmetries, emotion, and depression: conceptual and methodological conundrums. Psychophysiology 1998;35:607-614.

46. Keller J, Nitschke JB, Bhargava T, Deldin PJ, Gergen JA, Miller GA, et al. Neuropsychological differentiation of depression and anxiety. J Abnorm Psychol 2000;109:3-10.

47. Selvin S. Statistical analysis of epidemiologic data. Oxford: Oxford University Press;2004. 